\title{
D.F. Geary and F. Schaefer: Comprehensive paediatric nephrology
}

\author{
Mosby Elsevier editors, 2008
}

\author{
Sergio Fanconi
}

Received: 11 April 2009 / Accepted: 9 June 2009 /Published online: 10 July 2009

(C) Springer-Verlag 2009

This is an impressive textbook (1,099 pages) on paediatric nephrology from more than 120 contributors from varied specialities with a thorough international perspective; in fact, the two editors are from Toronto and Heidelberg, Germany, respectively, and the co-authors come from all over the world.

The 14 sections (tools of investigation, disorders of renal development, disorders of glomerular function, specific glomerular diseases, the kidney and systemic diseases, renal tubular disorders, tubulointestinal disease, urinary tract disorders, acute renal failure, hypertension, chronic kidney disease, renal replacement therapy, renal transplantation and renal pharmacology) cover in a concise and focused fashion almost every clinical condition. Comprehensive information delivers valuable insights, allowing informed and efficient decisions and easy access guidance. The index is of excellent quality, and the bibliography is extensive and covers the most important publications.

Clinical skills, diagnostic techniques, understanding pathogenesis and scientific advances, excellent imaging with multiple figures and schematic drawings (delivered also in a bonus CD ROM) and remarkable references to evidence based medicine are the backbones of the different chapters. An excellent chapter on traditional and complementary medicine is worth mentioning as it demonstrates the author's unbiased approach and their interest in all facets of renal problems.

This book is the ideal companion for paediatric nephrologists and urologists as well as trainees in nephrology and can be of great value for general paediatricians as a very rapid and informative source of knowledge for day-to-day care of their renal patients.
S. Fanconi $(\bowtie)$

CHUV,

Lausanne, Switzerland

e-mail: sergio.fanconi@chuv.ch 